\title{
Estimation of Snow Parameters with GPS and Galileo
}

\author{
Markus Lamm ${ }^{1}$, Franziska Koch ${ }^{2}$, Florian Appel $^{3}$, Patrick Henkel ${ }^{1}$ \\ ${ }^{1}$ ANavS GmbH - Advanced Navigation Solutions \\ Hessstrasse 36, 80798 Munich, Germany \\ Email: info@anavs.de \\ ${ }^{2}$ University of Natural Resources and Life Sciences (BOKU) \\ Institute of Water Management, Hydrology and Hydraulic Engineering \\ Muthgasse 18, 1190 Vienna, Austria \\ Email: franziska.koch@boku.ac.at \\ ${ }^{3}$ Vista Remote Sensing in Geosciences $\mathrm{GmbH}$ \\ Gabelsberger Str. 51, 80333 München, Germany
}

\begin{abstract}
The Snow Water Equivalent (SWE) is a key parameter for hydrological applications. In this paper, we describe a novel snow monitoring station that determines SWE solely from differential carrier phase measurements of both GPS and Galileo satellites. The SWE estimates are compared with several traditional sensors to demonstrate the achievable performance. The measurement results also show that just 4 Galileo satellites reduce the combined fixing time of RTK positioning by up to 3 minutes.
\end{abstract}

Keywords-Snow water equivalent, GPS, Galileo, SnowSense, Ambiguity fixing

\section{INTRODUCTION}

Hydropower plants optimize their power generation management with runoff predictions. These forecasts are based on meteorological models and in-situ measurements. A key parameter is the Snow Water Equivalent (SWE) that describes the amount of water being stored in snow.

There exist different sensors for measuring SWE, which are mainly based on weighing systems like snow pillows or scales [1]. However, they are vulnerable to measuring errors due to snow bridging. Also, a large flat foundation (typically $3 \mathrm{~m}$ by $3 \mathrm{~m}$ ) is required, making the installation expensive and complicated. SWE derivation based on gamma radiation or cosmic neutrons are up-coming techniques, but are very expensive and have difficulties to derive large amounts of SWE. Schmid et al. used a combination of an upward-looking Ground Penetrating Radar (upGPR) and a GPS receiver buried beneath the snowpack to derive SWE, LWC and snow height in [2]. Henkel et al. derived precise SWE information of dry snow solely from GPS signals in [3]. The restriction to GPS receivers is very attractive as it enables a substantial reduction of costs for the sensor and its installation.

The convergence time of GPS-based SWE estimation can be reduced by including other Global Navigation Satellite Systems. In this paper, we describe our novel SnowSense station that provides SWE information using both GPS and Galileo measurements.

\section{SnOw Monitoring Station}

In this section, we describe the snow-monitoring station with its components and the measurement principle. Fig. 1 shows an exemplary snow monitoring station in Millertown, Newfoundland, Canada.

\section{A. Station Components}

The station is equipped with the following components:

- mast system with rigging

- solar power unit

- Multi-GNSS and communication antennas

- measurement unit

- 2 Multi-GNSS receivers

- 1 processing module with processor for snow parameter estimation, power management unit and plug-in satellite communication module

- 1 solar charge controller

- 1 battery pack

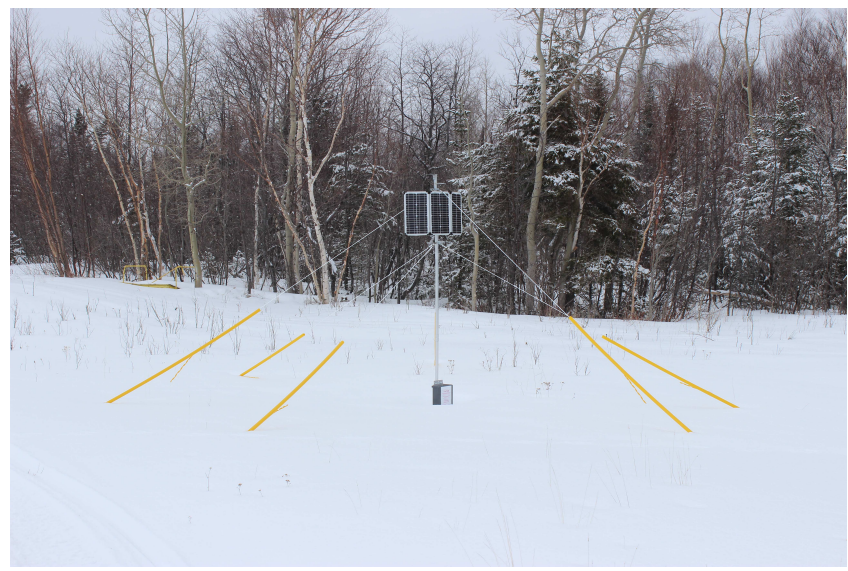

Figure 1. SnowSense station with solar power supply in Millertown in Newfoundland, Canada in April, 2018. 
Fig. 2 shows the processing board with the integrated snow parameter estimation, the power management unit and the Iridium satellite communication module.

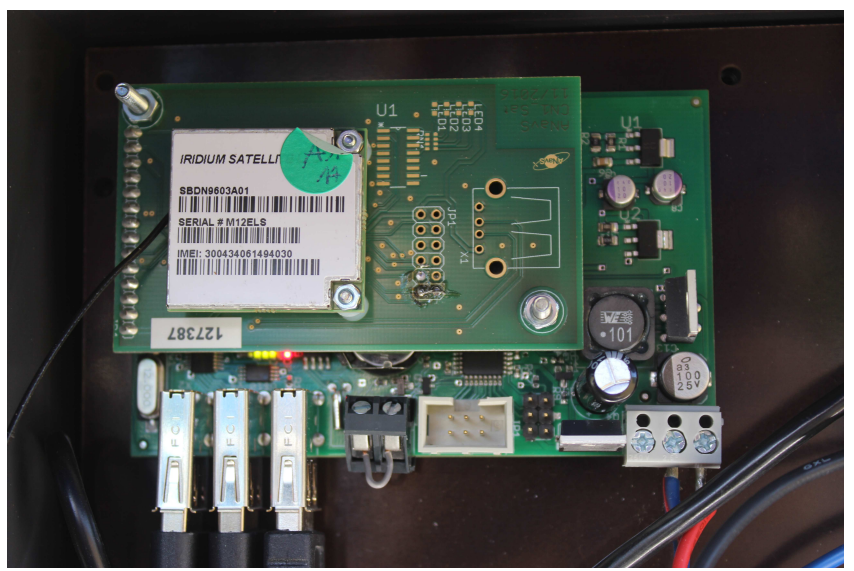

Figure 2. Processing board with integrated snow parameter estimation, power management unit and satellite communication module.

The use of solar power implies that only temporary measurements are possible. Therefore, we perform a power management according to the state diagram of Fig. 3. The measurement phase and sleep mode are the primary states. All other states are only transition states that occur if the battery voltage $V_{\mathrm{Bat}}$ or timing reach certain values.

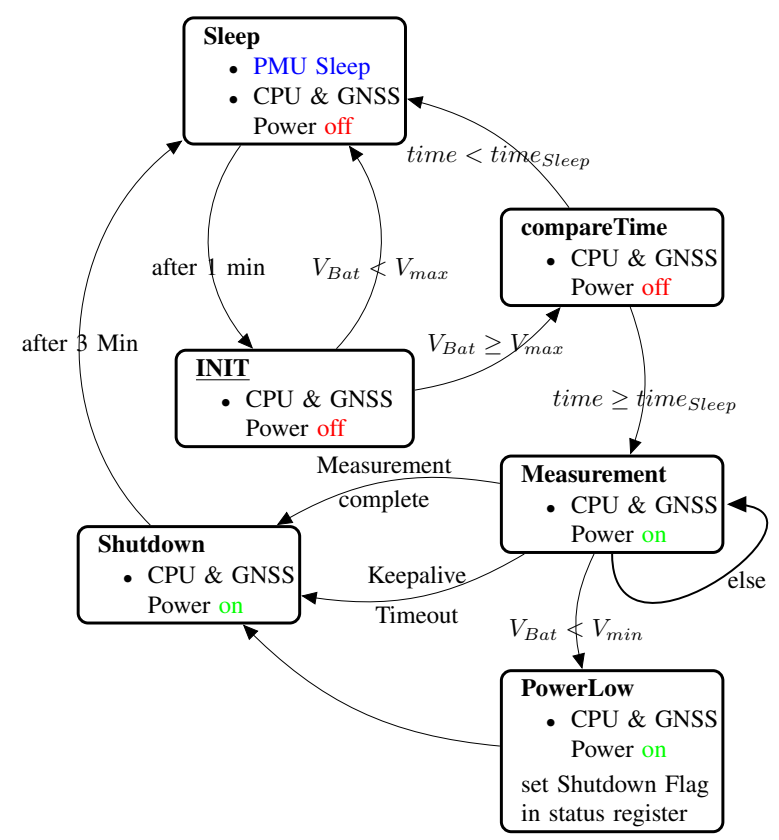

Figure 3. State diagram for Power Management Unit of SnowSense Station

\section{B. Differential Carrier-Phase GNSS Measurements}

Each snow monitoring station consists of two GNSS receivers: The antenna of the first GNSS receiver is placed on the ground and senses the snow through the delay and attenuation of the GNSS signal. The second GNSS antenna is placed on the mast above the snow and serves as reference antenna. This differential set-up was chosen to eliminate the following error sources by differencing the measurements of both GNSS receivers:

- tropospheric and ionospheric delays

- satellite position and clock offsets

- receiver and satellite phase biases and Earth tides

The elimination of this large variety of error sources enables a faster convergence and higher accuracy than the processing of absolute (i.e. undifferenced) measurements.

\section{Processing in two steps:}

Precise Positioning and Snow Parameter Estimation

We perform a processing in two sequential steps:

- calibration phase, i.e. estimation of relative position (3D baseline) between both GNSS receivers with millimeter-level accuracy in the absence of snow

- joint estimation of baseline, DD float ambiguities and pseudorange multipath errors with Kalman filter [4]

- fixing of float ambiguities to integers using the LAMBDA method [5] with hypothesis testing

- re-adjustment of baseline based on fixed ambiguities

- estimation of snow parameters

- least-squares estimation of SWE and float carrier phase ambiguity from time-series of DD phase measurements for each satellite pair using prior information on speed of light in snow and baseline estimate from previous step

- fixing of ambiguity estimates from float to integer numbers

- re-adjustment of SWE estimates

- combination of SWE estimates from different satellite pairs by least-squares adjustment

The first step refers to Real-Time Kinematic (RTK) positioning and is described in a variety of earlier publications, e.g. [4] and [6]. The focus of this paper is on the second step.

\section{Multi-GNSS instead of GPS-only measurements}

We use both GPS and Galileo instead of GPS-only measurements for the following reasons:

- increase of available carrier phase measurements compared to GPS-only measurements

- reliable ambiguity fix for RTK positioning and snow parameter estimation is achieved in shorter time with Multi-GNSS

- estimation of snow parameters is more accurate

Fig. 4 compares the fixing time of RTK positioning between GPS-only and combined GPS/ Galileo measurements performed at Pinakothek, Munich, Germany. There were 11 visible GPS and 4 visible Galileo satellites on May 25, 2018. As shown, Galileo reduces the fixing time by up to 3 minutes.

\section{Estimation of SNOW WATER Equivalent}

This section includes a precise model for snow-affected DD carrier phase measurements and the least-squares SWE estimation based on this model. 


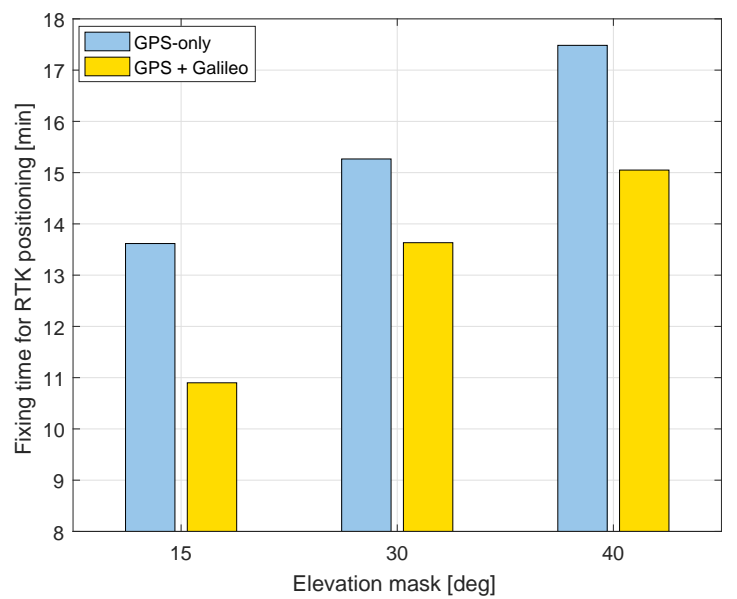

Figure 4. Fixing time for RTK positioning: The integration of Galileo reduces the ambiguity fixing time by up to 3 minutes. The number of satellites will further increase with the final deployment of Galileo.

\section{A. Measurement model}

The double difference (DD) carrier phase measurements between the receiver pair $u$ and $r$, the satellite pair $k \in$ $\{1, \ldots, K\}$ and $l$ (the latter one serving as reference satellite) of constellation $c \in\{1,2\}$ (GPS/ Galileo) is modeled according to [3] as

$$
\begin{aligned}
\lambda \varphi_{u r}^{k l, c}:= & \left(\lambda \varphi_{u}^{k, c}-\lambda \varphi_{r}^{k, c}\right)-\left(\lambda \varphi_{u}^{l, c}-\lambda \varphi_{r}^{l, c}\right) \\
= & \left(\vec{e}_{u}^{k, c}\right)^{\mathrm{T}}\left(\vec{x}_{u}-\vec{x}^{k, c}\right)-\left(\vec{e}_{r}^{k, c}\right)^{\mathrm{T}}\left(\vec{x}_{r}-\vec{x}^{k, c}\right) \\
& -\left(\vec{e}_{u}^{l, c}\right)^{\mathrm{T}}\left(\vec{x}_{u}-\vec{x}^{l, c}\right)+\left(\vec{e}_{r}^{l, c}\right)^{\mathrm{T}}\left(\vec{x}_{r}-\vec{x}^{l, c}\right) \\
& +\lambda N_{u r}^{k l, c}+\frac{c}{v_{\mathrm{s}}} m_{u}^{k l, c} \cdot \mathrm{SWE}_{u}+\varepsilon_{u r}^{k l, c},
\end{aligned}
$$

with the following notations:

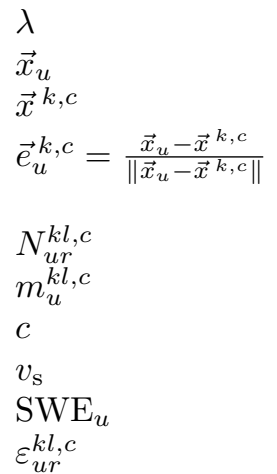

$\underset{\rightarrow}{\lambda}$

$\vec{x}^{k, c}$

wavelength

receiver position

satellite position

normalized direction vector

between satellite and receiver

DD integer ambiguity

differential mapping function

speed of light in vacuum

speed of light in snow

Snow Water Equivalent

DD phase noise including multipath

The speed of light $v_{\mathrm{s}}$ is assumed to be known in this paper and will be described in a follow-on paper.

The differential mapping function describes the mapping of the snow-induced vertical delay into slant direction, and is defined as

$$
m_{u}^{k l, c}:=m_{u}^{k, c}-m_{u}^{l, c}=\frac{1}{\sin \left(E_{u}^{k, c}\right)}-\frac{1}{\sin \left(E_{u}^{l, c}\right)},
$$

whereby $E_{u}^{k, c}$ is the elevation of the refracted satellite signal in snow. The satellite positions and the absolute position of the reference receiver are known, i.e. the DD measurements can be corrected for these parameters:

$$
\begin{aligned}
\lambda \tilde{\varphi}_{u r}^{k l, c}:= & \lambda \varphi_{u r}^{k l, c} \\
& -\left(\vec{e}_{u}^{k, c}\right)^{\mathrm{T}}\left(\vec{x}_{r}-\vec{x}^{k, c}\right)+\left(\vec{e}_{r}^{k, c}\right)^{\mathrm{T}}\left(\vec{x}_{r}-\vec{x}^{k, c}\right) \\
& +\left(\vec{e}_{u}^{l, c}\right)^{\mathrm{T}}\left(\vec{x}_{r}-\vec{x}^{l, c}\right)-\left(\vec{e}_{r}^{l, c}\right)^{\mathrm{T}}\left(\vec{x}_{r}-\vec{x}^{l, c}\right) \\
= & \left(\vec{e}_{u}^{k, c}\right)^{\mathrm{T}}\left(\vec{x}_{u}-\vec{x}_{r}\right)-\left(\vec{e}_{u}^{l, c}\right)^{\mathrm{T}}\left(\vec{x}_{u}-\vec{x}_{r}\right) \\
& +\lambda N_{u r}^{k l, c}+m_{u}^{k l, c} \frac{c}{v_{\mathrm{s}}} \cdot \mathrm{SWE}_{u}+\varepsilon_{u r}^{k l, c} \\
= & \left(\vec{e}_{u}^{k l, c}\right)^{\mathrm{T}} \vec{b}_{u r}+\lambda N_{u r}^{k l, c}+m_{u}^{k l, c} \frac{c}{v_{\mathrm{s}}} \cdot \mathrm{SWE}_{u}+\varepsilon_{u r}^{k l, c}
\end{aligned}
$$

with $\vec{b}_{u r}=\vec{x}_{u}-\vec{x}_{r}$ being the baseline vector between both GNSS receivers. The baseline is determined in the initial calibration phase, i.e. the respective term can be subtracted from the measurements:

$$
\begin{aligned}
\tilde{\lambda}_{u r}^{k l, c} & :=\lambda \tilde{\varphi}_{u r}^{k l, c}-\left(\vec{e}_{u}^{k l, c}\right)^{\mathrm{T}} \vec{b}_{u r} \\
& =\lambda N_{u r}^{k l, c}+m_{u}^{k l, c} \frac{c}{v_{\mathrm{s}}} \cdot \mathrm{SWE}_{u}+\varepsilon_{u r}^{k l, c} .
\end{aligned}
$$

The first term describes the carrier phase integer ambiguity, which is constant over time. The second term describes the snow-induced time delay, which changes with the satellite elevation during a satellite pass ( $\sim 6$ hours). We consider a time series of measurements to enable an estimation of both ambiguity and SWE. The measurements of different satellites are considered separately for integrity reasons, i.e. an erroneous time series of one satellite should not affect the SWE estimation of all other satellites. The time series of corrected DD carrier phase measurements of satellite pair $k l$ is obtained from Eq. (4) as:

$$
\begin{aligned}
\left(\begin{array}{c}
\tilde{\tilde{\varphi}} u_{u r}^{k l, c}\left(t_{1}\right) \\
\vdots \\
\tilde{\lambda} \varphi_{u r}^{k l, c}\left(t_{n}\right)
\end{array}\right) & =H\left(\begin{array}{c}
N_{u r}^{k l, c} \\
\operatorname{SWE}_{u}
\end{array}\right)+\left(\begin{array}{c}
\varepsilon_{u r}^{k l, c}\left(t_{1}\right) \\
\vdots \\
\varepsilon_{u r}^{k l, c}\left(t_{n}\right)
\end{array}\right), \\
H & =\left(\begin{array}{cc}
\lambda & \frac{c}{v_{\mathrm{s}}} m_{u}^{k l, c}\left(t_{1}\right) \\
\vdots & \vdots \\
\lambda & \frac{c}{v_{\mathrm{s}}} m_{u}^{k l, c}\left(t_{n}\right)
\end{array}\right) .
\end{aligned}
$$

\section{B. Estimation of Snow Water Equivalent}

We determine a float solution of the ambiguity and SWE by minimizing the sum of squared measurement residuals,

$$
\begin{aligned}
& \left(\begin{array}{c}
\hat{N}_{u r}^{k l, c} \\
\hat{S W E}_{u}^{k l, c}
\end{array}\right) \\
& =\min _{N_{u r}^{k l, c}, \mathrm{SWE}_{u}}\left\|\left(\begin{array}{c}
\tilde{\lambda}_{u r}^{k l, c}\left(t_{1}\right) \\
\vdots \\
\tilde{\lambda} \varphi_{u r}^{k l, c}\left(t_{n}\right)
\end{array}\right)-H\left(\begin{array}{c}
N_{u r}^{k l, c} \\
\mathrm{SWE}_{u}
\end{array}\right)\right\|_{\Sigma^{-1}}^{2} \\
& =\left(H^{\mathrm{T}} \Sigma^{-1} H\right)^{-1} H^{\mathrm{T}} \Sigma^{-1}\left(\begin{array}{c}
\tilde{\lambda}_{u r}^{k l, c}\left(t_{1}\right) \\
\vdots \\
\tilde{\lambda}_{u r}^{k l, c}\left(t_{n}\right)
\end{array}\right),
\end{aligned}
$$




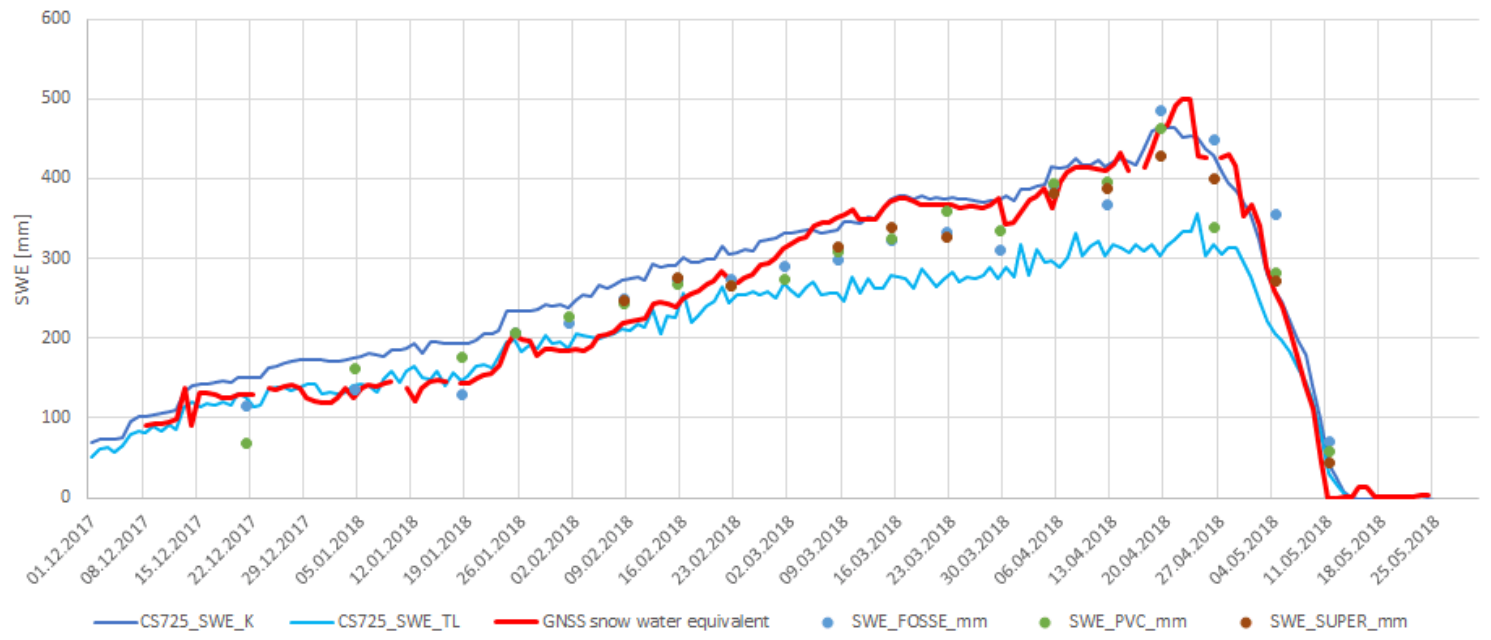

Figure 5. Comparison of SWE retrieval with GPS, gamma radiation sensors and manual measurements at MOMO in Canada during the winter 2017/18.

with $\Sigma$ being the covariance matrix of the measurements. We have added the satellite indices at the SWE estimate to highlight that it was determined solely based on measurements of one satellite pair $k l$.

The float ambiguity estimate is rounded to the nearest integer and the SWE estimate is adjusted accordingly, i.e. the ambiguity-fixed SWE is given by:

$$
\begin{aligned}
\mathrm{SWE}_{u}^{k l, c}= & \mathrm{SWE}_{u}^{k l, c} \\
& -\frac{\sigma_{\mathrm{SWE}_{u}^{k l, c}, \hat{N}_{u r}^{k l, c}}^{k l,}}{\sigma_{\hat{N}_{u r}^{k l, c}}^{k l,}}\left(\hat{N}_{u r}^{k l, c}-\left[\hat{N}_{u r}^{k l, c}\right]\right),
\end{aligned}
$$

with $\sigma_{\mathrm{SWE}_{u}^{k l, c}, \hat{N}_{u r}^{k l, c}}$ being the covariance between SWE and float ambiguity estimates, $\sigma_{\hat{N}^{k l, c}}^{2}$ being the variance of the float ambiguity estimate, and $[\cdot]$ being the rounding operator to the nearest integer number.

Finally, the ambiguity-fixed SWE estimates of all satellite pairs from both constellations are combined to one single SWE estimate:

$$
\mathrm{SW} E_{u}=\arg \min _{\mathrm{SWE}}\left\|\left(\begin{array}{c}
\mathrm{SWE}_{u}^{1} \\
\mathrm{SWE}_{u}^{2}
\end{array}\right)-\left(\begin{array}{c}
1^{K_{1}} \\
1^{K_{2}}
\end{array}\right) \mathrm{SWE}\right\|_{\Sigma_{\mathrm{SWE}}^{-1}}^{2},
$$

where $K_{c}$ denotes the number of visible satellites for constellation $c, 1^{K_{c}}$ is a column vector with $K_{c}$ ones, $\Sigma_{\mathrm{SWE}}$ is the SWE covariance matrix and $\mathrm{SWE}_{u}^{c}$ includes the SWE estimates from all double differences of constellation $c$, i.e.

$$
\mathrm{SWE}_{u}^{c}=\left(\mathrm{SLE}_{u}^{1 l, c}, \ldots, \mathrm{SWE}_{u}^{K_{c} l, c}\right)^{\mathrm{T}}
$$

The solution of the minimization (9) is given for a diagonal covariance matrix by

$$
\mathrm{SWE}_{u}=\left(\sum_{c=1}^{2} \sum_{k=1}^{K_{c}} \frac{1}{\sigma_{\mathrm{SWE}_{u}^{k l, c}}^{2}}\right)^{-1} \sum_{c=1}^{2} \sum_{k=1}^{K_{c}} \frac{\mathrm{SW}^{2} E_{u}^{k l, c}}{\sigma_{\mathrm{SWE}_{u}^{k l, c}}^{2}} .
$$

\section{Measurement Results}

The GNSS-based SWE estimation has been tested at the Forêt Montmorency (MOMO) test site $80 \mathrm{~km}$ north of Quebec City in Canada during the winter period 2017/18. The snow was mostly dry with temperatures as low as $-30^{\circ} \mathrm{C}$.

Fig. 5 shows that the GPS-based SWE information follows the manual measurements but is much less noisy. The CS725 SWE sensors (based on gamma radiation) differ by up to more than $100 \mathrm{~mm}$ from each other, and appear to be substantially biased. Our Multi-GNSS-based SWE system is very promising due to its accuracy, ecological friendliness and low cost.

\section{CONCLUSION}

In this paper, we presented our novel SnowSense station that measures the Snow Water Equivalent (SWE). The station includes two GNSS receivers, a solar power supply and an Iridium satellite communication for autonomous operations in remote locations. The performance of the GPS-based SWE estimation was validated at a meteorological test site in Quebec, Canada. We generalized the SWE estimation to include Galileo signals for an increased satellite number and faster processing, in particular in areas with limited satellite visibility.

\section{REFERENCES}

[1] D. Archer and D. Stewart, The installation and use of a snow pillow to monitor snow water equivalent, Water Environ. J., vol. 9, no. 3, pp. 221 - 230, 1995

[2] L. Schmid, F. Koch, A. Heilig, M. Prasch, O. Eisen, W. Mauser and J. Schweizer A novel sensor combination (upGPR-GPS) to continuously and nondestructively derive snow cover properties, Geophysical Research Letters, vol. 42, no. 9, pp. $3397-3405,2015$.

[3] P. Henkel, F. Koch, F. Appel, H. Bach, M. Prasch, L. Schmid, J. Schweizer and W. Mauser, Snow Water Equivalent of Dry Snow Derived From GNSS Carrier Phases, IEEE Transactions on Geoscience and Remote Sensing, vol. 56, iss. 6, pp. 3561 - 3572, Jun. 2018.

[4] P. Henkel and A. Sperl, Precise RTK Positioning with GPS/ INS Tight Coupling and Multipath Est., Proc. of ION ITM, pp. 1015 - 1023, 2016

[5] P.J.G. Teunissen, The least-squares ambiguity decorrelation adjustment: a method for fast GPS integer ambiguity estimation, Journal of Geodesy, vol. 70, pp. $65-82,1995$.

[6] H. Landau, U. Vollath and X. Chen, Virtual Reference Station Systems, Journal of Global Positioning Systems, vol. 1, no. 2, pp. $137-143,2002$. 